\title{
Über die Theorie der skiaskopischen Schattendrehung bei Astigmatismus.
}

\author{
Von \\ Dr. Alfred Borschke \\ in Wien
}

Für die Theorie der skiaskopischen Schattendrehung bei Astigmatismus mit schiefen Achsen gibt es zwei — vielleicht noch mehr Erklärungsmöglichkeiten.

Die eine sagt, dass infolge der verschiedenen Brechungsverhältnisse in den normalen Hauptachsen auch eine verschieden starke Vergrösserung in denselben stattfindet, dass also ein Quadrat, dessen Seiten den Hauptachsen parallel stehen, als ein Rechteck exscheinen muss, und daher eine Diagonale nicht mehr unter einem Winkel von $45^{0}$ erscheint. Wenn man hierbei noch berücksichtigt, dass gleichzeitig in einem Meridian ein aufrechtes, im andern ein verkehrtes Bild entworfen werden kann, ist die Möglichkeit vorhanden, sämtliche Stadien der Schattendrehung zu erklären und abzuleiten. Selbstverständlich gelten die derart entwickelten Gesetze nicht nur für den Spezialfall der Skiaskopie, sondern auch für jedes optische System, bei welchem ähnliche Bedingungen erfüllt sind.

Eine andere Erklärungsart, die aber der ersteren nicht etwa entgegengesetzt ist, sondern, wie wir sehen werden, im wesentlichen auf ganz dasselbe hinausläuft und ganz die gleichen Resultate liefern muss, ist folgende: In der kreisrunden Öffnung der Linsenkombination (der Pupille) sehen wir bei Verwendung von sphärischen Gläsern (einem anastigmatischen Auge) ein grösseres oder kleineres kreisrundes Stïck des Hintergrundes (der Netzhaut) in allen Dimensionen gleichmässig vergrössert oder verkleinert bis zur scheinbaren Grösse der kreisrunden Öffnung (der Pupille).

Bei Verwendung einer Linsenkombination mit Cylindergläsern (einem astigmatischen Auge) sehen wir in der kreisrunden Öffnung 
Über die Theorie der skiaskopischen Schattendrehung hei.Astigmatismus. 389

(der Pupille) ein den Sturmschen Graden entsprechendes elliptisches Stück ("Gesichtsfeld") des Hintergrundes, das aber in der kürzeren Achse stärker vergrössert erscheint als in der längeren, so dass die scheinbare Form dieses elliptischen Stückes ein Kreis wird, genau entsprechend dem Bild der Pupille.

Die erstere Art der Erklärung ungefähr (verschieden starke Vergrösserung in den rerschiedenen Achsen) gibt Weinhold in seiner Abbandlung: „Zur Theorie der skiaskopischen Schattendrehung bei Astigmatismus" ${ }^{ \pm}$), und es wäre nichts gegen diese Art der Erklärung einzuwenden, wenn sie entsprechend durchgeführt wäre und wenn Weinhold nicht die zweite Art der Erklärung (astigmatische "Verziehung des Gesichtsfeldes), welche ich bis zur genauen Konstruktion und Berechnung des Winkels der scheinbaren Schattengrenze ausgeführt habe ${ }^{2}$ ), abgelehnt hätte.

Wir wollen nun sehen, ob die Argumente, die Weinhold gegen die Erklärung durch astigmatische Verziehung des Gesichtsfeldes vorbringt, stichbaltig sind.

Bei der wohl meist gebräuchlichen Art zu skiaskopieren, bei der Verwendung eines Spiegels mit einem Loch, dessen Durchmesser erheblich kleiner ist als der Durchmesser der Pupille, ist das Gesichtsfeld auf der Netzhaut des Untersuchten gleich dem Zerstreuungsbilde des Spiegelloches. Ist nun aber das Spiegelloch grösser als die $\mathrm{Pu}-$ pille, oder blickt man am Rande des Spiegels vorbei, oder verwendet man zum Skiaskopieren planparallele Glasplatten ohne Spiegelbelag, so tritt an die Stelle des Spiegelloches ganz oder teilweise die Pupille des Beobachters. Das Gesichtsfeld ist dann natïrlich gleich dem Zerstreuungsbilde der Beobachterpupille, bzw. jener wirksamen Öffnung des untersuchenden Auges, das sich aus Spiegel und $\mathrm{Pu}-$ pillenrand kombiniert3). Ein solches Gesichtsfeld erfährt dann bei vorhandenem Astigmatismus des untersuchten Auges die gleiche Verzerrung wie im andern Fall das Zerstreuungsbild des Spiegelloches, und kaun diese Verzerrung in gleicher Weise zur Erklärung und Ableitung der Schattendrehung herangezogen werden.

Wir haben also gesehen, dass die Gründe, warum Weinhold die Erklärung des Phänomens der Schattendrehung durch die astig-

1) จ. Graefe's Arch. f. Ophthalm. Bd. LXII 2. S. 275.

2) Arch. f. Angenheilk. Bd. LII. S. 161.

3) Eine derartige Kombination habe ich S. 180-181 meiner Abhandlung (loc. cit.) beschrieben. 
matische Verziehung des Spiegellochbildes und damit des skiaskopischen Gesichtsfeldes nicht gelten lassen will, durchaus nicht stichhaltig sind. Es muss demnach zugegeben werden, dass eine solche Erklärung zulässig ist.

Sie erscheint aber auch zweckmässig, weil auf derselben Grundlage, dem Ineinandergreifen des Beleuchtungsfeldes und Gesichtsfeldes und den Beziehungen des letzteren zur Pupille, auch die andern Probleme der Skiaskopie sich erfolgreich behandeln lassen, und weil mit einfachen geometrischen Konstruktionen die scheinbare Schattenrichtung sich unschwer konstruieren oder nach einer aus der Konstruktion abgeleiteten Formel berechnen lässt.

Die Weinholdsche Darstellung bietet für diese Konstruktion keinen Ersatz. Weinhold kommt wohl zu dem Resultate, dass eine Schattendrehung stattfinden muss, "dass jeder bestimmten relativen Entfernung zwischen Hornhaut und Umschlagspunkt ein bestimmter Drehungswinkel entspricht". Hierbei übersieht jedoch Weinhold, dass bei einem astigmatischen Auge $z$ wei Umschlagspunkte vorbanden sind, für jede der beiden Hauptachsen je einer. Nach seiner Darstellung wäre der Umschlagspunkt eines und desselben astigmatischen Auges an verschiedener Stelle, je nachdem man es sich entstanden denkt aus der Kombination mit einem Konvexcylinder oder mit einem Konkavcylinder, dessen Achse auf der des ersteren senkrecht steht $\left.{ }^{1}\right)$. Der grösste Teil der Weinholdschen Abhandlung behandelt das Sehen durch eine einfache Cylinderlinse (wobei es sich allerdings nur um einen Umschlagspunkt handeln kann), während die optischen Verhältnisse bei einem astigmatischen Auge einer Kombination eines sphärischen Konvexglases mit einer Cylinderlinse entsprechen.

Schliesslich möchte ich noch, obwohl das eigentlich mit dem Thema der Schattendrehung nichts zu tun hat, auf einen Einwand zurückkommen, welchen mir Weinhold bezüglich des Gesichtsfeldes gemacht hat, dass sich nämlich Lichtquelle und Spiegel nie gleichzeitig auf der Netzhaut des Beobachteten scharf abbilden.

Die von Weinhold angeführten Tatsachen lassen sich natürlich nicht bestreiten; es handelt sich hierbei jedoch nicht um ein Versehen meinerseits, sondern um eine absichtliche Ausserachtlassung eines

1) Bekanntlich kann man ja jede Art von regulärem Astigmatismus ebenso gut durch Konvex- wie durch Konkavcylinder erzeugen, wenn man gleichzeitig das entsprechende sphärische Glas verwendet. 
Über die Theorie der skiaskopischen Schattendrehung bei Astigmatismus. 391

Faktors, dessen Besprechung an der betreffenden Stelle als mehr minder belanglos nur eine unnötige Komplikation bedeuten würde. Tatsächlich wird hierdurch das Gesichtsfeld in keiner Weise verändert, sondern nur die Breite des Beleuchtungsfeldes, die übrigens auch von dem entsprechenden Durchmesser der Lichtquelle abhängig ist, ein Umstand, den ich an gleicher Stelle ebenfalls vernachlässigen zu dürfen giaubte. An anderer Stelle, bei Besprechung der Genauigkeit der skiaskopischen Methode der Refraktionsbestimmung (S. 182) habe ich diese beiden Faktoren kurz erwähnt, und möge dies als Beweis dafür gelten, dass es sich an ersterwähnter Stelle nicht um ein Versehen meinerseits handeln kann. 